\section{Colonic embolisation: useful but caution required}

The value of diagnostic visceral angiography in the investigation and management of acute and chronic gastrointestinal bleeding is well established, its use in this role having been first described over 35 years ago. ${ }^{1}$ It is possible to identify the source of haemorrhage in $87 \%$ of patients actively bleeding at the time of the study, and to identify a lesion which could be responsible for the problem in $74 \%$ of those not bleeding at the time of investigation. ${ }^{2}$ These figures are dependent on a number of factors, including the use of meticulous angiographic technique, the quality of the imaging equipment and the experience of the operator in both the acquisition and interpretation of data. ${ }^{3}$

In a patient thought to be actively bleeding at the time of the angiogram, the vessel most likely to be responsible is examined first - for example, the inferior mesenteric artery (IMA) in rectal bleeding, the coeliac axis in haematemesis. If a source is not found then the other major visceral vessels are studied in turn. If the superior mesenteric artery (SMA) and IMA studies appear normal in a case of bleeding thought clinically to be of lower gastrointestinal origin, the coeliac axis should also be studied, not only because there may be aberrant vascular anatomy (the coeliac axis and its tributaries can give rise to middle colic and jejunal arteries), but also because bleeding from the upper gastrointestinal tract may present in an unusual way and should be excluded. ${ }^{14}$ Portal hypertension, for instance, may be best revealed on a coeliac study. In the patient not actively bleeding it is vital to examine all three major vessels, the coeliac axis, SMA and IMA, with selective and superselective studies being performed as appropriate.

Therapeutic vascular embolisation in the management of gastrointestinal bleeding was first described by Bookstein et al in $1974 .^{5}$ They used autologous blood clot in nine cases, controlling the bleeding in the three patients with haemorrhage from the lower gastrointestinal tract. Embolisation procedures require the operator to achieve superselective catheterisation of the vessel or vessels involved in the pathological process to maximise the efficacy of the manoeuvre and minimise the risk of complications, such as ischaemic necrosis or the inadvertent embolisation of neighbouring structures. All potential routes of collateral blood supply need to be examined and embolised where necessary. Advances in catheter and guidewire technology (e.g. effective co-axial catheter systems and hydrophilic guidewires) have made it increasingly possible to manipulate catheters into small and tortuous vessels and this has greatly increased the safety and efficacy of the method. Embolic agents have also been refined and minute embolisation coils are available which can be delivered through very fine coaxial catheter systems. ${ }^{6}$ These advances have revived interest in the use of embolisation in the lower gastrointestinal tract, ${ }^{7}$ formerly an area in which the technique was regarded as hazardous owing to the relative paucity of the collateral blood supply in some areas.

Rosenkrantz et al in $1982^{9}$ described three cases of colonic mucosal necrosis in a total of 23 patients embolised and this incidence of serious complications has deterred many radiologists from using the technique in the lower bowel. Their paper and subsequent publications deserve greater scrutiny. It is important to look at the figures in the context of the underlying clinical condition and in comparison with the success and complications associated with alternative treatment-for example, vasopressin infusion, or emergency partial or total colectomy.

In their review of the literature, Guy and colleagues ${ }^{7}$ found reported morbidity and mortality rates for emergency hemicolectomy of $50 \%$ and $30 \%$, respectively. Vasopressin infusion achieved haemostasis in up to $90 \%$ of cases, but with a rebleeding rate of $20 \%$ and an associated complication rate as high as $43 \%$. Complications included myocardial infarction, cardiac arrhythmia, cardiorespiratory arrest, and visceral infarction. In patients treated by therapeutic embolisation, colonic infarction and ischaemia was reported in up to $20 \%$ of cases. In their own series, nine patients underwent successful embolisation. There were no cases of procedurally related colonic infarction and only two cases of endoscopically diagnosed asymptomatic mucosal ischaemia. They attributed their success to advances in catheter and guidewire technology enabling highly selective catheterisation thus minimising the area of tissue embolised.

In a recently published series, Gordon and colleagues ${ }^{8}$ reviewed 17 patients considered for embolisation. In three cases they did not achieve a highly selective catheter position and therefore did not proceed. In 13 (93\%) of the remaining 14 cases haemostasis was achieved. There were no episodes of recurrent bleeding and no evidence of bowel ischaemia or stricture formation either endoscopically or clinically on follow up (mean 10 months). In all cases microcoils were delivered through fine coaxial catheters. The authors emphasised the need for highly selective catherisation, but advised against the use of fine particulate emboli which may occlude mucosal capillaries.

The series reported in this issue (see page 79) by Nicholson et al adds further weight to the growing evidence that with meticulous technique and modern equipment, embolisation is an effective treatment in acute lower gastrointestinal haemorrhage with a comparatively low major complication rate.

It is important to emphasise a number of issues which affect the safety and success of embolisation procedures. Clinical liaison is paramount. Careful patient selection is vital for embolisation, the technique being reserved for those subjects in whom conservative management has failed, and emergency surgery is contemplated. A high order of technical expertise is essential and an appropriate range of catheters, guidewires and embolic agents should be readily available. Adequate follow up is essential not only to detect complications such as stricture development, but also to determine the underlying pathology responsible for the haemorrhage if this is not already known. 
Embolisation is not the treatment of choice for an operable colonic carcinoma!

Finally, embolisation may in many cases represent definitive treatment, but in others may usefully convert an emergency colectomy carrying a high risk in a desperately sick patient to an elective, more limited, resection in a stable patient. The inclusion of vascular intervention from 1 April 1998 in NCEPOD will bring all vascular interventional procedures under close scrutiny, a development welcomed by active interventionists who wish to maintain and raise the standards of their discipline.

A P HEMINGWAY

D J ALLISON

Department of Imaging,

Hammersmith Hospitals NHS Trust,

Hammersmith Hospital,

Du Cane Road,

London W12 OHS, UK
1 Nussbaum M, Baum S, Blakemore W, et al. Demonstration of intraabdominal bleeding by selective arteriography. $\mathcal{F} A M A 1965 ; 191: 117-18$.

2 Allison DJ, Hemingway AP, Cunningham DA. Angiography in gastrointestinal bleeding. Lancet 1982;ii:30-3.

3 Allison DJ. Gastrointestinal angiography. In: Grainger R, Allison DJ, eds. Diagnostic radiology. A textbook of medical imaging. 3rd edn. Edinburgh: Churchill Livingstone, 1997:1091-108.

4 Zuckerman DA, Bocchini TP, Birnbaum EH. Massive haemorrhage in the lower gastrointestinal tract in adults: diagnostic imaging and intervention. AfR Am f Roentgenol 1993;161:703-11.

5 Bookstein JJ, Chlosta EM, Foley D, et al. Transcatheter hemostasis of gastrointestinal bleeding using modified autogenous clot. Radiology 1974;113:277-85.

6 Morse SS, Clark RA, Puffenbarger A. Platinum microcoils for therapeutic embolization: non-neuroradiological application. AfR Am $f$ Roentgenol 1990;155:401-3.

7 Guy GE, Shetty PC, Sharma RP, et al. Acute lower gastrointestinal haemorrhage: treatment by superselective embolization with polyvinal alcohol particles. AfR Am f Roentgenol 1992;159:521-6.

8 Gordon RL, Ahl KL, Kerlan RK, et al. Selective arterial embolization for the control of lower gastrointestinal bleeding. AfR Am f Roentgenol 1997;174: $24-8$.

9 Rosenkrantz H, Bookstein JJ, Rosen RJ, et al. Post embolic colonic infarction. Radiology 1982;142:47-51.

\section{Life (and death) in the Fas lane}

The hallmark of an immune response is the generation of a large new cohort of specific lymphocytes dedicated to the removal of the stimulating antigen. The protection afforded by the mobilisation of an army of potentially destructive cells comes at a price as these cells, if uncontrolled, may turn on the host. Thus when excessive immune activity occurs, immunopathology resulting from the "friendly fire" of inflammatory cells may compromise healthy tissue. To safeguard against this, mechanisms have evolved that remove the expanded population of leucocytes both at the end of the immune response, when antigen is eradicated, and also when excessive activation may be taking place. ${ }^{1}$ The ligation of a surface membrane molecule CD95 (Fas/Apo-1) on activated T cells by its ligand leads to apoptosis. ${ }^{2}$ Although once activated, the expression of the CD95 receptor is stable, the expression of CD95 (Fas)-ligand, which is also found on T cells, is transient and occurs after recent $\mathrm{T}$ cell activation. Thus when there are too many recently activated $\mathrm{T}$ cells in close proximity to each other, death rather than continued stimulation will occur, and this contributes to the downsizing of the local activated lymphocyte pool.

Although the expression of the CD95-ligand is largely (but not completely) restricted to activated T lymphocytes, many different cell types can express the CD95 receptor molecule. ${ }^{2}$ The paper by Ueyama et al in this issue (see page 48) shows convincingly that there is high CD95ligand expression by $\mathrm{T}$ cells in lesions of ulcerative colitis but not Crohn's disease. This, together with the observations that the CD95 receptor is expressed by epithelial cells in ulcerative colitis and apoptotic cell numbers are increased in colonic epithelia in some inflammatory bowel diseases, ${ }^{3}$ suggests that CD95/CD95-ligand interactions may be involved in the pathophysiology of ulcerative colitis. Although this is an attractive hypothesis, some caution is needed when interpreting the results. Although cells may show a high level of CD95 receptor expression, they are not necessarily susceptible to CD95 mediated death. ${ }^{4}$ In analogy, the presence of a gun is not harmful per se, it has to be loaded first. In order to be sensitive to triggering by the CD95 pathway, the appropriate downstream death signal- ling molecules have to be in place in the cytoplasm. ${ }^{4}$ For this reason, the presence of CD95 on epithelial cells in ulcerative colitis, which is only weakly expressed, does not necessarily mean that these cells will die upon contact with the ligand on $\mathrm{T}$ cells. One way in which to approach this in future studies would be to correlate the extent of apoptosis in the colonic epithelium of patients with ulcerative colitis with the local presence of $\mathrm{T}$ cells expressing CD95-ligand. Another way forward would be to compare the expression of CD95 and susceptibility to CD95 induced death in in vitro cultured gut epithelial cells from patients with ulcerative colitis and Crohn's disease.

Although further investigation into the induction of apoptosis in the epithelium in patients with ulcerative colitis is clearly warranted, one should not lose sight of the central question raised in Ueyama et al's paper. As the CD95-ligand is transiently expressed on activated T cells, the presence of this molecule in ulcerative colitis but not Crohn's disease is suggestive of recent, extensive $\mathrm{T}$ cell activation in the gut. What is the stimulus for the $\mathrm{T}$ cell activation in ulcerative colitis? The possibilities include normal tissue elements, suggesting a possible autoimmune defect as the basis of this disease, ${ }^{5}$ or foreign antigen/ infectious agents. It is possible that the cells may even be proliferating in situ, a point which also requires further investigation. Irrespective of the ongoing stimulus, the intense $\mathrm{T}$ cell activation would potentially bring into play CD95/CD95-ligand associated homoeostatic mechanisms directed against the prevention of $\mathrm{T}$ cell overstimulation. ${ }^{5}$ Thus it may be also be possible to detect apoptotic $\mathrm{T}$ cells in these lesions. One unfortunate by-product of this normal control mechanism may be the non-specific, "bystander destruction" of the CD95 positive epithelium by cells expressing the CD95-ligand, as suggested by Ueyama et al. If subsequent studies confirm that this is the mechanism by which epithelial cell damage occurs in ulcerative colitis, then therapeutic strategies targeting this apoptosis inducing pathway may have to be considered.

Department of Clinical Immunology,

A N AKBAR

Royal Free Hospital School of Medicine,

Rowland Hill Street,

London NW3 2PF, UK

email:akbar@rfhsm.ac.uk 
1 Lenardo $M$. Fas and the art of lymphocyte maintenance. $f$ Exp Med 1966;183:721-4.

1966;183:721-4.
Krammer PH, Behrmann I, Daniel P, et al. Regulation of apoptosis in the immune system. Curr Opin Immunol 1994;6:279-86.

3 Lee FD. Importance of apoptosis in the histopathology of drug related lesions in the large intestine. F Clin Pathol 1993;46:118-22.
4 Peter ME, Kischkel FC, Scheuerpflug CG, et al. Resistance of cultured peripheral $\mathrm{T}$ cells towards activation-induced cell death involves a lack of recruitment of FLICE (MACH/Caspase 8) to the CD95 death-inducing signalling complex. Eur f Immunol 1997;27:1207-12.

5 Akbar AN, Salmon M. Cellular environments and apoptosis: tissue microenvironments control activated T cell death. Immunol Today 1997;18:72-6.

\section{Call for Patients with Familial Pancreatic Disease: The EUROPAC Register}

We are establishing a national UK register (EUROPAC) of families with hereditary pancreatitis, familial pancreatic cancer and where pancreatic cancer has occurred as part of a familial cancer syndrome. This collaboration in Liverpool is between the Department of Clinical Genetics ( Dr Ian Ellis) and the Academic Department of Surgery (Professor John Neoptolemos). The data and samples are collected by behalf of ESPAC (the European Study Group for Pancreatic Cancer), Professor Markus Büchler, Berne, and Professor Hans Beger, Ulm. The study will collaborate with Dr David Whitcomb of the Midwest Multicenter Pancreatitis study group in the United States. We aim to recruit families who are prepared to donate blood for DNA studies. We hope to gain a clearer understanding of the genetic relationship between hereditary pancreatitis and familial pancreatic cancer, and develop screening protocols for individuals at risk.

Hereditary pancreatitis is associated with a mutation in the recently identified cationic trypsinogen gene. This mutation renders the enzyme active within the pancreas, leading to autodigestion. Individuals with recurrent pancreatitis have a greatly increased risk of developing pancreatic cancer, and there is some evidence that DNA analysis of cells from pancreatic fluid may be valuable in detecting premalignant changes which can predict the development of pancreatic adenocarcinoma.

The criteria for inclusion in the study are as follows:

- Hereditary pancreatitis: Three relatives with chronic pancreatitis in the absence of ethanol dependence, hypercalcaemia, or an obstructive cause.

- Familial pancreatic cancer: Two first degree relatives with pancreatic adenocarcinoma. Three or more relatives with pancreatic ductal adenocarcinoma. Pancreatic ductal adenocarcinoma in any two relatives where the sum of their ages is less than 110 years.

- Other familial cancer syndromes: A single documented pancreatic ductal adenocarcinoma in any family with an established familial cancer syndrome-for example, BRCA2, FAMMM, A-T, HNPCC, or FAP.

If you know of any suitable families who may be interested in joining the study, please contact: Fiona McRonald, Clinical Genetics, Alder Hey Children's Hospital, Eaton Road, Liverpool L12 2AP. Tel: 01512525905.

Thank you for your help. 